Regular Paper

\title{
Lab-on-Chip System Combined Optical Tweezers and Dielectrophoresis
}

\author{
Yoshihiro MIZUTA*1 and Kozo TAGUCHI*1
}

\begin{abstract}
Cell manipulation and operation have played important roles in modern biotechnology, hence a number of researchers have developed them during the past decades. In this paper we introduce two techniques based on dielectrophoresis (DEP) and optical tweezers: Distinction between viable cells and non-viable cells using DEP, Manipulating cells captured by positive DEP (pDEP) using optical tweezers. And then we suggest a system combined both DEP and optical tweezers, that would be a useful tool in biotechnology. This system will be useful to perform cell operation and other applications in biotechnology.
\end{abstract}

Keywords: optical tweezers, dielectrophoresis, yeast, lab-on-chip system.

(Received: 24 July 2014, Revised: 7 May 2015)

\section{Introduction}

Biotechnology and cell technology have been developed in modern society, this field is raising expectations for new technology. In particular, applications of cell are being researched by a lot of research institution such as biosensor and cell operation. And then researchers are pursuing more smaller and simple device for being low cost. In this background, a variety of techniques that can control cells are generated such as using magnetic, electric and other methodologies. In 1970th, Ashkin discovered force that could capture microparticles when light irradiated it in focus point, and then he noticed gradient force and proposed basics of optical tweezers [1]. This technique realized manipulating micro particles in three-dimensinal space. In recently decade, optical tweezers is also applied fusion cell as the ways how to realize efficient cell fusion [2].

When dielectric particles are subjected to AC field, they move and array to electrodes. In 1950th, Pohl called this phenomena dielectrophoresis (DEP) [3]. Since at that time, theory of DEP has been investigated by several experiments and are applied applications in biological systems. DEP can manipulate particles using DEP force that are occurred when particles is placed in a non-uniform electric field, so that are used in biological applications such as separation, trapping and sorting [4-6].

In this paper we introduce two techniques based on DEP and optical tweezers: Distinction between viable cells and non-viable cells using DEP, Manipulating cells captured by positive DEP (pDEP) using optical tweezers. DEP was performed by Au-thin film that made by easily ways. About manipulating cells captured by DEP using optical tweezers, we also examined relationship between

\footnotetext{
Correspondence: Kozo Taguchi, Department of Electrical and Electronic Engineering, Ritsumeikan University, Kusatsu, Shiga, Japan, 525-8577

Email: taguchi@se.ritsumei.ac.jp

${ }^{*}$ Ritsumeikan University
}

voltage applied on the two electrodes of the DEP and the laser power output of the optical tweezers. And then we would suggest a system combined both DEP and optical tweezers that would be a useful tool in biotechnology. This system will be useful to perform cell operation and other applications in biotechnology.

\section{Principle}

\subsection{Optical tweezers}

Optical tweezers apply to manipulate microparticle and single cell with non-invasive and non-contact. It is important for biological objects [1]. There are mainly two types in optical tweezers, the first is method using microscope objective lens, and second is using optical fiber [7]. Both of them are the same principle of using momentum preservation. As shown in Fig. 1 (a), laser focused by objective lens irradiates micro object. When refractive index of micro object is higher than that of medium surrounding, ray 1 is refracted at surface of object and changes direction. Thus momentum of ray is changed at surface. According to the change in momentum, a force F1a works in surface of object. As well as $\mathrm{F} 1 \mathrm{~b}$ is worked at place that ray 1 goes out of object. Therefore F1 works in center of object as resultant force, and as well as F2 is works by ray 2 . As a result radiation pressure $\mathrm{F}$ works upward, the object was trapped in the point that this power, buoyancy and gravitation are balanced. When object also veers pivot of laser as shown in Fig. 1 (b), pressure F finally works in direction of focus and the object is trapped in around focus point.

And then, model of optical tweezers using fiber are described by Fig. 1 (c). As shown in Fig. 1 (c), micro object is irradiated by an optical fiber. The transverse force Ftr is known to restoring forces from experiments [7]. Micro object is trapped in the point where the optical force FtrH and optical force FaxH, are precisely balanced. 
(a)

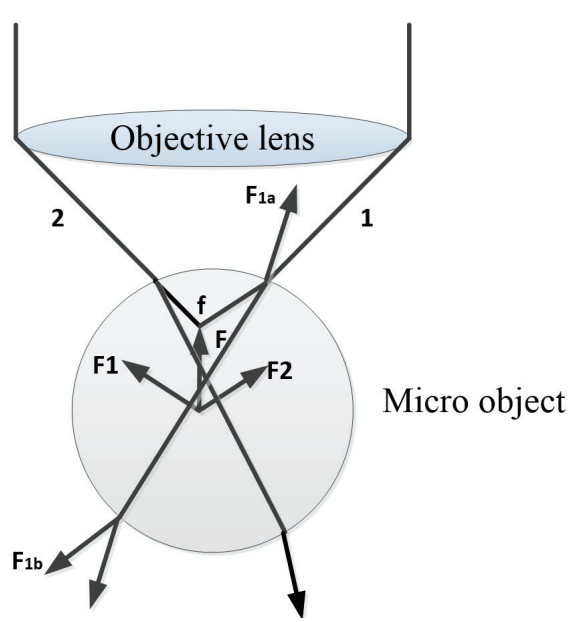

(b)

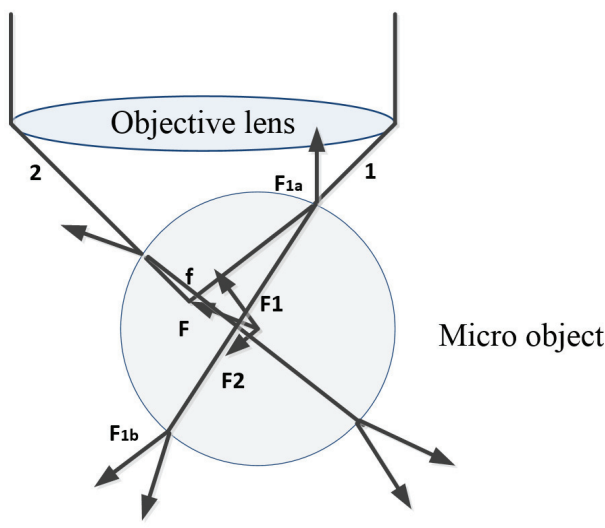

(c)

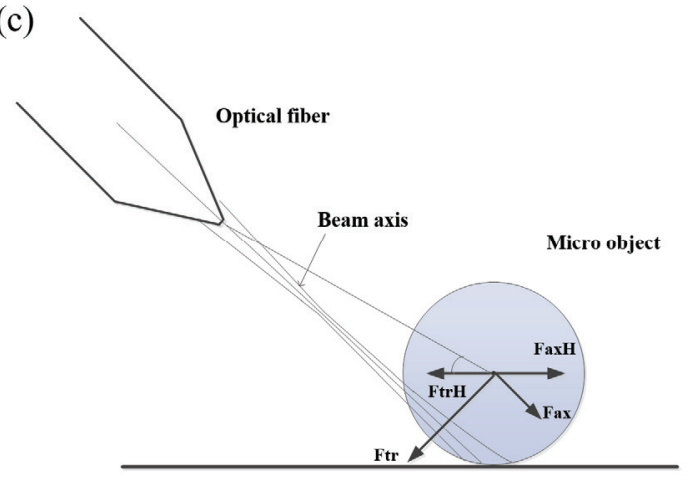

Fig. 1. Optical tweezers.

Next we would think measuring radiation pressure. Considering radiation pressure, trapping power and trapping efficiency $\mathrm{Q}$ are important. These are known to the define as

$$
\begin{aligned}
& F=6 \pi \mu a v \\
& F=Q P n / c
\end{aligned}
$$

where $F$ is the trapping power, $\mu$ is viscosity coefficient, $a$ is radius of object, $v$ is velocity, $Q$ is the trapping efficiency, $P$ is average laser power, $n$ is the refractive index of surrounding medium and $c$ is the speed of light in the free space [8]. $F$ is found in giving (1) by measuring $v$ in experiment and $Q$ is founded by substituting the $F$ for (2).

\subsection{Dielectrophoresis}

As shown in Fig. 2, dielectric particle moves and forms pearl chain between two electrodes with nonuniform AC field. We call this phenomenon DEP, this technique is used to manipulate cell in biomechanics. Some dielectric particles show two movement types by changing frequency of the applied electric field. Dielectric particles move toward the regions of strong electric field and attach to electrode in pDEP as shown in Fig. 2 (a), while move toward the regions of low electric field and repel electrode in negative DEP (nDEP) as shown in Fig. 2(b).
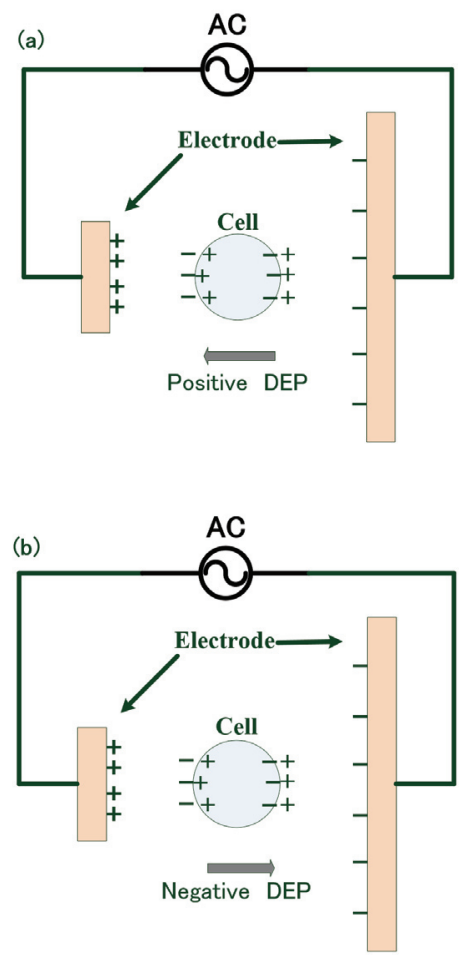

Fig. 2. (a) Dielectric particle move to the strong field in pDEP. (b) Dielectric particle repel from the strong field in $\mathrm{nDEP}$

According to theory investegaed by reserchers [4$6,9]$, DEP force is difined as

$$
F_{D E P}=2 \pi r^{3} \varepsilon_{m} \operatorname{Re}[K(\omega)] \cdot \nabla E^{2}
$$


where $r$ is radius of dielectric particle, $\varepsilon_{m}$ is dielectric constant of suspended medium, $E$ is non-uniform electric field, $\operatorname{Re}[\mathrm{]}$ shows the real part, and $K(\omega)$ is factor of Clausius-Mossotti, which is defined as

$$
K(\omega) \equiv\left[\frac{\varepsilon_{p}^{*}-\varepsilon_{m}^{*}}{\varepsilon_{p}^{*}+2 \varepsilon_{m}^{*}}\right]
$$

where $\varepsilon_{p}$ and $\varepsilon_{m}$ are the dielectric constant, the asterisk (*) shows that dielectric constant is complex quantity. That $\varepsilon^{*}$ is defined as

$$
\varepsilon^{*} \equiv \varepsilon-i \frac{\sigma}{\omega}
$$

where $\sigma$ is conductivity, $\omega$ is angular frequency of the electric field, $\varepsilon$ is dielectric constant that is complex quantity.

Hence, $K(\omega)$ and $\nabla E^{2}$ are important factors in DEP, $K(\omega)$ is depend on frequency of the electric field and characteristic of dielectric constants of particle and medium. When $K(\omega)$ is positive, pDEP is generated, while $K(\omega)$ is negative, $\mathrm{nDEP}$ is generated. DEP force is proportional to the field strength, and then $\nabla E^{2}$ are related to pattern of electrodes, which lead to generate DEP force in effective. A lot of patterns of electrode are reported such as parallel paired electrodes, a planar quadrupole microelectrode $[4,10]$.

\section{Materials}

\subsection{Yeast cells}

In this experiment, yeast cells (BY4741 S.cerevisiae) were used in DEP. Cells is almost spherical and has diameter in the range of 5 to $10 \mu \mathrm{m}$. Non-viable cells of yeasts are prepared for separation system. Non-viable cells were obtained by a heat treatment of 80 degrees Celsius in 10 minutes. And they are stained with methylene blue. Methylene blue works only for non-viable cells, while viable cells maintain non-stained.

\subsection{DEP chip}

To perform DEP, microelectrode was fabricated by coating $\mathrm{Au}$ thin film on the thin glass slide as shown in Fig. 3 [10]. This way is easy to make microelectrode and uses just simple devices. The thin glass slide was covered by adhesive tape except rectangle pattern, and then that was coated $\mathrm{Au}$ thin-film by deposition of ion coater (IB-3 Eiko). In order to add microfluidic system, we attached silicone rubber to the thin grass slide coated Au thin-film.

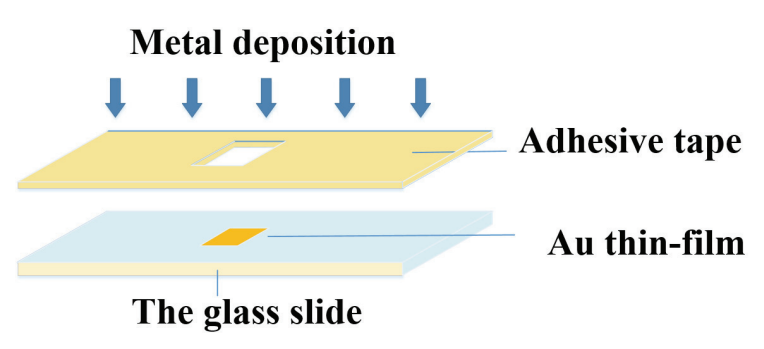

Fig. 3. Au thin-film.

\subsection{Optical tweezers operation}

For microparticle manipulation, we assembled the optical tweezers setup. We used laser diode at $980 \mathrm{~nm}$ (SNO534289, Lumics, Germany). It was focused using microscope immersion objective lens $(100 \times / 1.25$, Edmund, USA). For measuring trapping power and trapping efficiency, we used the motorized translation stage (KT-LS13-M, Zaber Technologies Inc, Canada). We prepared microsphere $(10 \mu \mathrm{m})$ and yeast (Saccharomyces cerevisiae) as target microparticle.

We checked movement of optical tweezers with microsphere and yeast. We measured trapping power and trapping efficiency as shown in Table 1 and 2. Target yeast and microsphere was trapped using focused laser beam with $30 \mathrm{~mW}$ average laser power.

Table 1 Measuring trapping power and trapping efficiency of Microsphere.

\begin{tabular}{|l|r|}
\hline \multicolumn{2}{|c|}{ Trapping power of microsphere $(\mathrm{F}=\mathbf{6} \boldsymbol{\pi} \boldsymbol{\mu a v})$} \\
\hline \hline $\mathrm{F}:(\mathbf{N})$ & $\mathbf{1 . 6 8 4 2 5 E - 1 1}$ \\
\hline$\pi$ & 3.14159265 \\
\hline$\mu: v i s c o s t y$ of water $(\mathrm{Pa} \cdot \mathrm{s})$ & 0.000893521 \\
\hline a:radius of microsphere $(\mathrm{m})$ & 0.000005 \\
\hline v:velocity $(\mathrm{m} / \mathrm{s})$ & 0.0002 \\
\hline
\end{tabular}

\begin{tabular}{|l|r|}
\hline \multicolumn{2}{|c|}{ Trapping efficie ncy of micros phe re $(\mathbf{F}=\mathbf{Q P n} / \mathbf{c})$} \\
\hline \hline Q: & $\mathbf{0 . 1 2 6 5 4 7 5 6 6}$ \\
\hline F:trapping power $(\mathrm{N})$ & $1.68425 \mathrm{E}-11$ \\
\hline P:average laser power $(\mathrm{W})$ & 0.03 \\
\hline $\mathrm{c}:$ speed of light $\left(\mathrm{m}^{\wedge} \mathrm{s}^{\wedge}-1\right)$ & 299792485 \\
\hline n:refractive index of water & 1.33 \\
\hline
\end{tabular}


Table 2 Measuring trapping power and trapping efficiency of Yeast.

\begin{tabular}{|l|r|}
\hline \multicolumn{2}{|l|}{ Trapping power of Yeast $(\mathrm{F}=\mathbf{6} \pi \boldsymbol{\mu a v})$} \\
\hline \hline $\mathrm{F}:(\mathrm{N})$ & $\mathbf{1 . 4 2 0 6 \mathrm { E } - 1 2}$ \\
\hline$\pi$ & 3.14159265 \\
\hline$\mu:$ viscosty of water $(\mathrm{Pa} \cdot \mathrm{s})$ & 0.001004866 \\
\hline a:radius of yeast $(\mathrm{m})$ & 0.0000025 \\
\hline v:velocity $(\mathrm{m} / \mathrm{s})$ & 0.00003 \\
\hline
\end{tabular}

\section{Trapping efficie ncy of Ye ast $(\mathrm{F}=\mathrm{QPn} / \mathrm{c})$}

\begin{tabular}{|l|r|}
\hline \hline Q: & $\mathbf{0 . 0 1 0 6 7 3 7 8 2}$ \\
\hline F:trapping power $(\mathrm{N})$ & $1.4206 \mathrm{E}-12$ \\
\hline P:average laser power $(\mathrm{W})$ & 0.03 \\
\hline $\mathrm{c}:$ speed of light $\left(\mathrm{m}^{*} \mathrm{~s}^{\wedge}-1\right)$ & 299792485 \\
\hline n:refractive index of water & 1.33 \\
\hline
\end{tabular}

\section{Experiment}

\subsection{Distinction between viable cells and non- viable cells using DEP}

In this experiment, we construct a lab-on-chip system which can easily perform distinction between viable cell and non-viable cell. Some distinction between viable cells and non-viable cells of using DEP was reported in biotechnology.
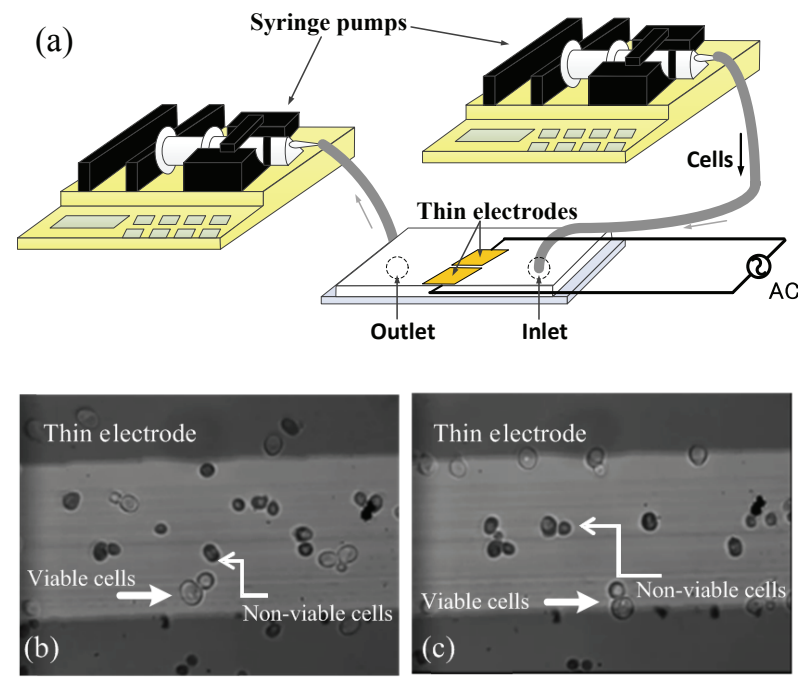

Fig. 4. (a)Lab-on-chip system equipped thin electrode and flow separation. (b)Include viable cells(white cells) and non-viable cells stained with methylene blue(black cells). (c)Only viable cells attached to the electrodes.

We made lab-on-chip equipped thin electrodes and flow separation as shown in Fig. 4 (a). The lab-on-chip could perform pDEP with viable yeast cells in frequency range $300 \mathrm{kHz}$ to $15 \mathrm{MHz}$, and they formed pearl chain. While non-viable didn't show behaviour in frequency range $300 \mathrm{kHz}$ to $15 \mathrm{MHz}$. So we could perform distinction between viable cells and non-viable cells using DEP.

First, we carried out pDEP experiment with yeast cells including both viable cells and non-viable cells stained with methylene blue. And then only viable cells attached to the electrodes. Second, we washed nonviable yeast cells away with syringe pumps applied 100 $\mu \mathrm{l} / \mathrm{m}$. The results are shown in Fig. 4 (b) and (c). Viable cells keep attaching to the electrodes, while non-viable cells are flowed into syringe pump.

\subsection{Manipulating cells captured by pDEP using optical tweezers}

In the second section, we moved cells captured by pDEP using the optical tweezers. To manipulate cells attached electrode by pDEP would take advantage of applications such as cell isolation and fusion. And we examined relationship between voltage applied on the two electrodes of the DEP and the laser power output of the optical tweezers. According to the theory of DEP, DEP force is known to be in proportional to the applied voltage of the DEP. Trapping power of optical tweezers also turns strong when laser power output is increase. In optical tweezers, we used laser diode at $980 \mathrm{~nm}$ and laser was focused by a microscope immersion objective lens as shown in Fig. 5 (a). In DEP, we made lab-on-chip equipped microelectrodes. First we carried out pDEP to yeast cells, the applied voltage was $0.5,1.0$ and $1.5 \mathrm{~V}$ peak to peak, and frequency of $500 \mathrm{kHz}$. Second we repeled viable cell from the electrode of the DEP using the optical tweezers, as shown in Fig. 5 (b). The result of our experiment clearly showed that the laser power of the optical tweezers was increased gradually when more and more voltage was applied, as shown in Fig. 5 (c). These observations appear to match the theoretical formula of DEP force.

\section{Result\&Discussion}

Using DEP and optical tweezers, we could perform distinction between viable cells and non-viable cells and repel cells captured by DEP as mentioned above. DEP could distinct between viable cells and non-viable cells easily. Optical tweezers could manipulate only viable cells at will. Combing the two experiment of using DEP and optical tweezers, we suggested new biochip. The lab-on-chip has functions of distinction, fusion and other applications in easily. We will try to perform the Lab-on-chip system with not only yeast cells but also other types of cell in the future. In fact, we are discussing such experiment with protoplast of plant. Protoplasts of plant are almost spherical and have diameter in the range of 15 to $60 \mu \mathrm{m}$. Protoplasts of red cabbage and carrot were observed pDEP in our lab-on-chip as shown in Fig. 6 (a) and (b). Additionally we measured limits 
speeds of protoplasts. Size of protoplasts is variety about 10 to $40 \mu \mathrm{m}$ as shown in Table 3 .
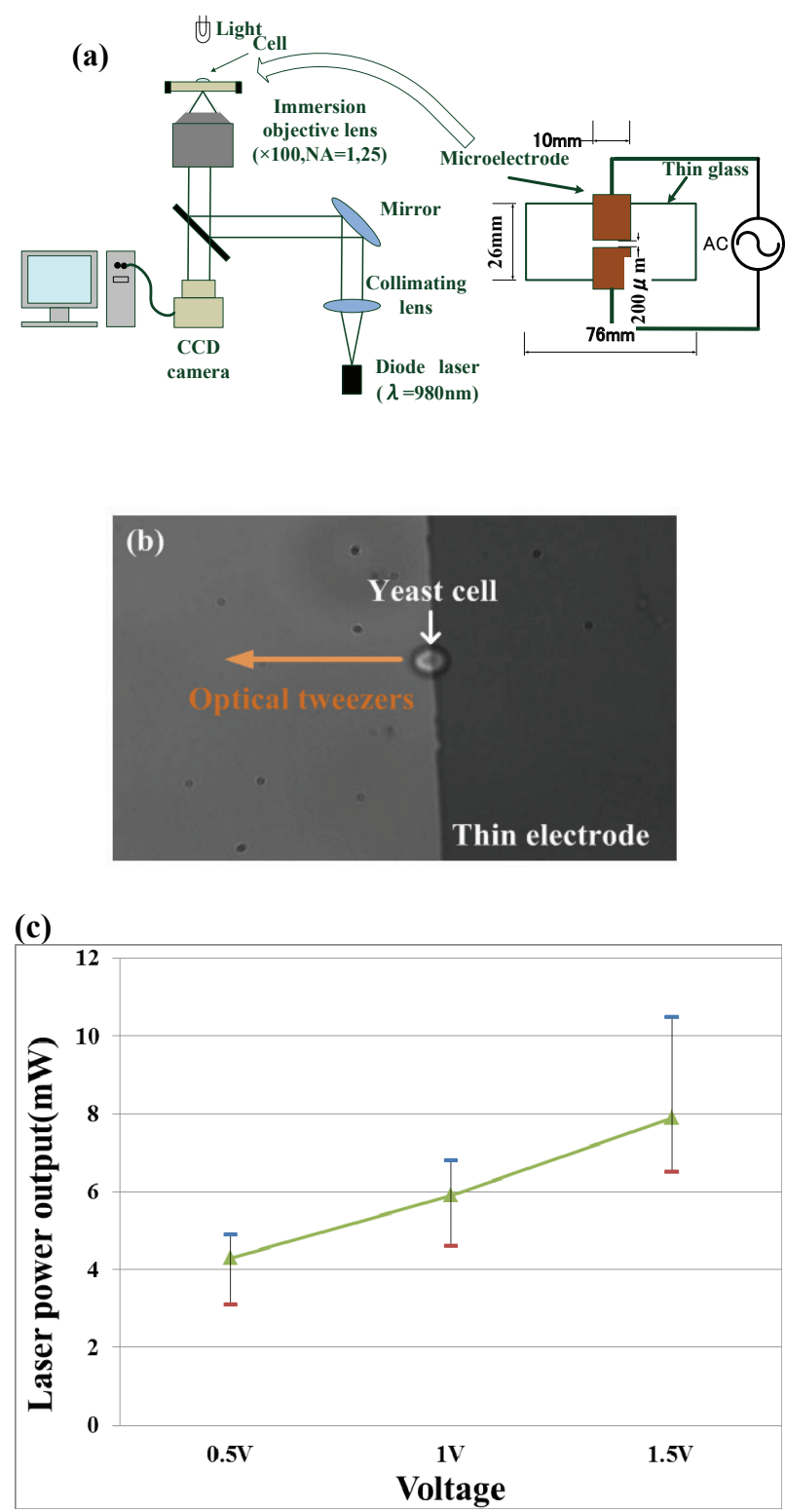

Fig. 5. (a) Combining optical tweezers and DEP experimental setup. (b) Illustration of repelling yeast cell using optical tweezers. (c) Relationship between voltages that function generator supplies to DEP and laser power output.

\section{Conclusion}

According to this measuring, our optical tweezers experiment could perform manipulating protoplasts of plant cells, moreover we were discussing possibility of distinction between viable cell and non-viable cell using DEP chip we suggested in easily. From this study, we are deeply engaged in the lab-on-chip of DEP and optical tweezers, and then make lab-on-chip system that is applied to other cells without yeast cells. For example, firstly we carried out distinction of using DEP, there are only activity cells, and we can remove non-viable cells. Then that activity cells are manipulated by optical tweezers of being low power, after that we could perform cell operation, cell transfection and fusion changing high power. So lab-on-chip system equipped both DEP and optical tweezers would be valid in cell applications.
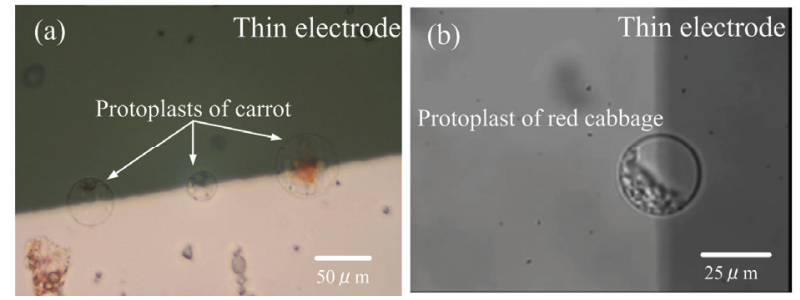

Fig. 6. (a) Protoplasts of carrot are attached to electrode by pDEP. (b) Protoplasts of red cabbage are attached to electrode by $\mathrm{pDEP}$.

Table 3 The limits of velocity of protoplasts.

\begin{tabular}{|l|r|r|r|r|}
\hline Diame ter of protoplast $(\mu \mathrm{m})$ & 14.5 & 20.5 & 27.2 & 36.2 \\
\hline Limit of velocity $(\mu \mathrm{m} / \mathrm{s})$ & 50 & 40 & 30 & 10 \\
\hline
\end{tabular}

\section{Acknowledgement}

The authors would like to thank Prof. Wakayama. The yeast cells were supplied from Enzyme Technology Lab of Ritsumeikan Univ.

\section{References}

[1] Ashkin, "Forces of a single-beam gradient laser trap on a dielectric sphere in the ray optics regime," Biophysical Jounal, Vol.61, pp.569-582, 1992.

[2] R. W. Steubing, S. Cheng, W.H. Wright, Y. Numajiri and M.W. Berns, "Laser induced cell fusion in combination with optical tweezers: The laser cell fusion trap," Cytometry, Vol.12, No.6, pp.505-510, 1991.

[3] Herbert A. Pohl and Joe S. Crane, "Dielectrophoresis of Cells," Biophysical Jounal, Vol.11, No.9, pp.711-727, 1971.

[4] H. Morgan, M. P. Hughes and N. G. Green, "Separation of Submicron Bioparticles by Dielectrophoresis," Vol.77, No.1, pp.516-525, 1999.

[5] W.J. Liu, J. Zhang, L.J.Wan, K.W. Jiang, B.R. Tao, H.L. Li,W.L. Gong and X.D. Tang, "Dielectrophoretic manipulation of nano-materials and its application to micro/nano-sensors," Vol.133, No.2, pp.664-670, 2008.

[6] S. Bhattacharya, T.-C. Chao, A. Ros, N. Ariyasinghe, R. Ros, Y. Ruiz, D. Lake, N. Ariyasinghe and R. Ros, "Selective trapping of single mammalian breast cancer cells by insulator-based dielectrophoresis," Vol.406, No.7, pp.1855-1865, 2014. 
[7] K. Taguchi, H. Ueno, T. Hiramatsu and M. Ikeda, "Optical trapping of dielectric particle and biological cell using optical fibre," Electronics Letters, Vol.33, No.27, pp.413 414, 1997.

[8] H.Felgner, O. Muller and M. Schliwa, "Calibration of light forces in optical tweezers," Vol.34, pp.977-982, 1995.

[9] C. Zhang, K. Khoshmanesh, A. Mitchell and K. Kalantarzadeh, "Dielectrophoresis for manipulation of micro/nano particles in microfluidic systems," Vol.396, pp.401-420, 2010.

[10] K. Nishimoto and K. Taguchi, "A Simplified Fabrication Method of Dielectrophoresis Chip Using Au Thin-Film and Box Cutter," Advanced Materials Research, Vol. 909, pp. 58-62, 2014. 